\title{
On Betti Numbers of Complement of Hyperplanes
}

By

\author{
Hiroaki Ter $\Lambda 0^{*}$
}

\section{§1. Introduction}

Let $X$ be a non-void finite family of hyperplanes in $\mathbb{C}^{n+1}$ or $\mathbb{P}^{n+1}(\mathbb{C})$. Denote $\boldsymbol{P}^{n+1}(\boldsymbol{C})$ simply by $\boldsymbol{P}^{n+1}$. By $|X|$ denote we the union of all hyperplanes belonging to $X$. In this article we give some formulas (Theorem A, B) for computing the Betti numbers of $\mathbb{C}^{n+1}|| X \mid$ or $\mathbb{P}^{n+1}|| X \mid$.

Define a set

$$
L(X)=\left\{\bigcap_{H \in A} H ; A \subset X\right\} \cup\left\{\text { the ambient space }\left(\mathbb{C}^{n+1} \text { or } \mathbb{P}^{n+1}\right)\right\} \backslash\{\varnothing\}
$$

and introduce a partial order $\succ$ into $L(X)$ by

$$
s>t \Longleftrightarrow s \subset t \quad(s, t \in L(X)) .
$$

If $L(X)$ has a unique maximal element, then $X$ is said to be central. In other words, $X$ is central if and only if $\cap_{H \in X} H \neq \varnothing$.

Recall the following

(1.1) Definition. The Möbius function $\mu: L(X) \rightarrow \mathbb{Z}$ is inductively defined by

$$
\begin{aligned}
& \mu(0)=1, \\
& \mu(s)=-\sum_{\substack{t \geq s \\
t \neq s}} \mu(t),
\end{aligned}
$$

where 0 stands for the ambient space (the minimal element in $L(X)$ ).

By $r(s)$ w'e denote the length of the longest chain in $L(X)$ below $s(s \in L(X))$.

In this article we call a non-void finite family of hyperplanes in $\mathbb{C}^{n+1}$ (or $\boldsymbol{P}^{n+1}$ ) an affine (resp. projective) $n$-arrangement. Then we have

(1.2) Theorem A. Let $X$ be an affine (or projective) n-arrangement.

Communicated by S. Nakano, September 16, 1980.

* Department of Mathenatics, International Christian University, Mitaka 181, Japan. Partially supported by Kakenhi No. 574047. 
Then the Poincaré polynomial of $\boldsymbol{C}^{n+1}|| X \mid$ (resp. $\left.\boldsymbol{P}^{n+1}|| X \mid\right)$ equals

$$
\begin{gathered}
\sum_{s \in L(X)} \mu(s)(-t)^{r(s)} \\
\text { (resp. } \left.\sum_{s \in L(X)} \mu(s)\left\{(-t)^{r(s)}-(-t)^{n+2}\right\} /(1+t)\right) .
\end{gathered}
$$

Remark. When $X$ is central affine, this result was proved by Orlik-Solomon [1] (5.2). Moreover they explicitly determined the graded $\boldsymbol{C}$-algebra structure of $H^{*}\left(\boldsymbol{C}^{n+1}|| X \mid, \boldsymbol{C}\right)$. There it was also announced, without proof, that their method would go well in case that $X$ is a non-central affine arrangement.

We will define the freeness of any affine (or projective) arrangement and the generalized exponents of a free affine (resp. projective) arrangement in Section 4 (resp. Section 3). All of the definitions are given via the case of a free central affine arrangement studied in [3] [4] [5].

The following theorem gives another formula for the Betti numbers of $\boldsymbol{C}^{n+1} \backslash|X|$ (or $\boldsymbol{P}^{n+1}|| X \mid$ ) by using the generalized exponents:

(1.3) Theorem B. Let $X$ be a free affine (or projective) $n$-arrangement and $\left(d_{0}, \ldots, d_{n}\right)$ be its generalized exponents. Then the Poincaré polynomial of $\boldsymbol{C}^{n+1} \backslash|X|\left(\right.$ resp. $\left.P^{n+1} \backslash|X|\right)$ equals

$$
\prod_{i=0}^{n}\left(1+d_{i} t\right)
$$

Remark. This result was obtained in [5] when $X$ is central affine. Our proof is nothing other than the reduction to the case.

The following Sections 2, 3 and 4 are devoted to the proofs of Theorems $A$ and B. Section 2 is for the central affine case, Section 3 for the projective case, and Section 4 for the (non-central) affine case.

\section{§2. The Central Affine Case}

In this section we briefly review some known results on a central affine $n$-arrangement $X$.

By an appropriate coordinate change we can assume that $\cap_{H \in X} H$ contains the origin $\mathbf{0}$ of $\boldsymbol{C}^{n+1}$. Let $Q \in \boldsymbol{C}\left[z_{0}, \ldots, z_{n}\right]$ be a defining equation of $X$, that is, $V(Q)=|X| . \quad$ By $\mathcal{O}$ denote we $\mathcal{O}_{\boldsymbol{C}^{n+1}, \boldsymbol{0}}$. Then

$D(X):=\{\theta$; a germ at the origin of holomorphic vector field such that

$$
\theta \cdot Q \in Q \cdot \mathcal{O}\}
$$


is an $\mathcal{O}$-module. We call $X$ to be free if $D(X)$ is a free $\mathcal{O}$-module.

Assume that $X$ is free. Let $\left\{\theta_{0}, \ldots, \theta_{n}\right\}$ be a free basis for $D(X)$ such that each $\theta_{i}$ is homogeneous of degree $d_{i}$ (see [3] 2.10). Then we call the integers $\left(d_{0}, \ldots, d_{n}\right)$ the generalized exponents of $X$. They depend only on $X$ (see [3] 2.12).

Throughout this article $b_{i}(S)$ stands for the $i$-th Betti number of a topological space $S$ for any integer $i\left(b_{i}(S)=0\right.$ if $\left.i<0\right)$.

The following "trinity" was proved in [1] (5.2) and [5] (Main Theorem):

Theorem $\mathbf{A}, \mathbf{B}$ (central affine version).

$$
(-1)^{i} \sum_{\substack{s \in L(X) \\ r(s)=i}} \mu(s)=b_{i}\left(\mathbb{C}^{n+1} \backslash|X|\right)=\pi_{i}\left(d_{0}, \ldots, d_{n}\right)
$$

for any integer $i$, where $\pi_{i} \in \mathbb{Z}\left[t_{0}, \ldots, t_{n}\right]$ is the elementary symmetric polynomial of degree $i\left(\pi_{i}=0\right.$ if $i<0$ or $\left.i>n+1\right)$.

\section{§3. The Projective Case}

Let $X(\neq \emptyset)$ be a projective $n$-arrangement. Let $Q \in \mathbb{C}\left[z_{0}, \ldots, z_{n+1}\right]$ be a homogeneous polynomial defining $|X| \subset \mathbb{P}^{n+1}$. Then there exists a central affine $(n+1)$-arrangement $\tilde{X}$ such that

$$
V(Q)=|\tilde{X}| \subset \mathbb{C}^{n+2}
$$

\section{(3.1) Proposition.}

$$
b_{i}\left(\boldsymbol{P}^{n+1} \backslash|X|\right)+b_{i-1}\left(\boldsymbol{P}^{n+1} \backslash|X|\right)=b_{i}\left(\mathbb{C}^{n+2}|| \tilde{X} \mid\right)
$$

for any integer $i$.

Proof. Consider the natural projection

$$
\pi: \mathbb{C}^{n+2}|| \tilde{X}\left|\longrightarrow \mathbb{P}^{n+1}\right||X|,
$$

then this is a $\mathbb{C}^{*}$-bundle. So we have the Gysin exact sequence

$$
\begin{aligned}
\cdots \longrightarrow & H^{q}\left(\mathbb{P}^{n+1}|| X \mid\right) \stackrel{\pi^{*}}{\longrightarrow} H^{q}\left(\mathbb{C}^{n+2} \backslash|\tilde{X}|\right) \longrightarrow H^{q-1}\left(\mathbb{P}^{n+1} \backslash|X|\right) \\
& \longrightarrow H^{q+1}\left(\mathbb{P}^{n+1} \backslash|X|\right) \stackrel{\pi^{*}}{\longrightarrow} \cdots .
\end{aligned}
$$

What we have to prove is the injectivity of each $\pi^{*}$ above.

Let $\varphi$ be a rational $q$-form on $\mathbb{P}^{n+1}$ whose pole is only along $|X|$. Assume that $\pi^{\cdot} \varphi=d \eta$ for some homogeneous rational $(q-1)$-form $\eta$ on $\mathbb{C}^{n+2}$ with pole only along $|\tilde{X}|$, where $\pi^{\circ} \varphi$ means the pull-back of $\varphi$ by $\pi$.

Then there exists a rational $(q-1)$-form $\psi$ on $\mathbb{P}^{n+1}$ with pole only along 
$|X|$ such that

$$
\pi \cdot \psi=-\left\langle\theta, \frac{1}{\operatorname{deg} Q} \frac{d Q}{Q} \wedge \eta\right\rangle .
$$

Here $\langle\theta$,$\rangle stands for the contraction with the Euler vector field$

$$
\theta=\sum_{i=0}^{n+1} z_{i}\left(\partial / \partial z_{i}\right)
$$

Then we call show

$$
d \psi=\varphi
$$

by a direct but lengthy computation (or by applying (2.6), (2.7) and (2.9) in [2]).

These facts imply that each $\pi^{*}$ is injective and thus (3.1). Q.E.D.

For any integer $i$, we have

$$
b_{i}\left(\boldsymbol{P}^{n+1} \backslash|X|\right)=\sum_{j=0}^{i}(-1)^{i-j} b_{j}\left(\boldsymbol{C}^{n+2} \backslash|\tilde{X}|\right)
$$

in the light of (3.1).

Define an injective mapping

$$
\rho: L(X) \longrightarrow L(\tilde{X})
$$

by $\rho(s)=\left(\right.$ the closure of $\pi^{-1}(s)$ in $\left.C^{n+2}\right)(s \in L(X))$, where $\pi$ is the natural projection: $\boldsymbol{C}^{n+2} \backslash\{\boldsymbol{0}\} \rightarrow \boldsymbol{P}^{n+1}$. Then it is easy to see that

$$
r(\rho(s))=r(s), \quad \text { and } \quad \mu(\rho(s))=\mu(s), \quad(s \in L(X)) .
$$

Notice that $\operatorname{im} \rho \supset\{t \in L(\tilde{X}) ; r(t)<n+2\}$. Thus we have

$$
\begin{aligned}
b_{i}\left(\boldsymbol{P}^{n+1} \backslash|X|\right) \\
=\sum_{j=0}^{i}(-1)^{i-j} b_{j}\left(\boldsymbol{C}^{n+2} \backslash|\tilde{X}|\right) \quad(\text { by }(3.2)) \\
=(-1)^{i} \sum_{j=0}^{i} \sum_{\substack{t \in L(\tilde{X}) \\
r(t)=j}} \mu(t) \\
=(-1)^{i} \sum_{j=0}^{i} \sum_{\substack{s \in L(X) \\
r(s)=j}} \mu(s)
\end{aligned}
$$

for $i<n+2$. It is obvious that

$$
b_{i}\left(\boldsymbol{P}^{n+1} \backslash|X|\right)=0 \quad \text { if } \quad i \geqq n+2 .
$$

Thus a brief computation leads us to Theorem A (projective version).

(3.4) Definition. We call $X$ to be free if $\tilde{X}$ is free. 
Assume that $X$ is free. Let $\left(d_{0}, d_{1}, \ldots, d_{n}\right)$ be the generalized exponents of $\tilde{X}$, then we can assume that $d_{0}=1$ (due to the existence of the Euler vector field) because $\tilde{X} \neq \varnothing$. The generalized exponents of $X$ are defined by $\left(d_{1}, \ldots, d_{n}\right)$.

For any integer $i$, we have

$$
\begin{aligned}
b_{i} & \left(\boldsymbol{P}^{n+1} \backslash|X|\right) \\
& =\sum_{j=0}^{i}(-1)^{i-j} b_{j}\left(\boldsymbol{C}^{n+2} \backslash|\tilde{X}|\right) \quad(\text { by }(3.2)) \\
& =\sum_{j=0}^{i}(-1)^{i-j} \pi_{j}\left(1, d_{1}, \ldots, d_{n}\right) \\
& =\pi_{i}\left(d_{1}, \ldots, d_{n}\right),
\end{aligned}
$$

where $\pi_{j}$ 's $(j \leqq i)$ are the elementary symmetric polynomials of degree $j$ (with $n$ or $(n+1)$-variables). This proves Theorem B (projective version).

\section{§4. The (Non-Central) Affine Case}

Let $H_{\infty}$ be a hyperplane in $\mathbb{P}^{n+1}$, then we can identify $\mathbb{C}^{n+1}$ with a Zariski open $\boldsymbol{P}^{n+1} \backslash H_{\infty}$ of $\boldsymbol{P}^{n+1}$. Let $X$ be a (perhaps non-central) affine $n$-arrangement. Define a projective $n$-arrangement

$$
X_{\infty}=X \cup\left\{H_{\infty}\right\} .
$$

We can regard $L(X)$ as a subset of $L\left(X_{\infty}\right)$ by a correspondence

$$
s \longmapsto \text { the closure of } s \text { in } \mathbb{P}^{n+1}
$$

$(s \in L(X))$. Put $L=L(X)$ and $L_{\infty}=L\left(X_{\infty}\right)$. By $L(i)$ (or $L_{\infty}(i)$ ) we denote a set

$$
\{t \in L ; r(t)=i\} \quad\left(\text { resp. }\left\{t \in L_{\infty} ; r(t)=i\right\}\right)
$$

for any integer $i$.

Define

$$
M(s):=\{t \in L ; r(t)=i-1, t \prec s\}
$$

for any $s \in L_{\infty}(i) \backslash L$. By $\mu$ we denote the Möbius function on $L_{\infty}$. Then wc have

(4.1) Lemma. Let $i<n+2$, then

1) $L(i-1)=\bigcup_{s \in L_{\infty}(i) \backslash L} M(s) \quad$ (disjoint),

2) $\mu(s)=-\sum_{t \in M(s)} \mu(t) \quad$ for any $s \in L_{\infty}(i) \backslash L$.

Pronf. 1): For any $t \in L(i-1)$, we have 


$$
r\left(t \cap H_{\infty}\right)=i, t \in M\left(t \cap H_{\infty}\right) \text {, and } t \cap H_{\infty} \in L_{\infty}(i) \backslash L .
$$

This implies that

$$
L(i-1)=\underset{s \in L L_{\infty}(i) \backslash L}{\bigcup} M(s) .
$$

Next assume that $t \in M(s)\left(s \in L_{\infty}(i) \backslash L\right)$, then $t \cap H_{\infty} \prec s$ and $r(s)=i$ $=r\left(t \cap H_{\infty}\right)$. Thus $s=t \cap H_{\infty}$, which implies that

$$
M(s) \cap M\left(s^{\prime}\right)=\varnothing \quad\left(s \neq s^{\prime}, s, s^{\prime} \in L_{\infty}(i) \backslash L\right) .
$$

2): We prove by an induction on $i$. When $i \leqq 0, L_{\infty}(i) \backslash L=\varnothing$. So 2) holds true trivially. Let $s \in L_{\infty}(i) \backslash L$ and $k<i$. Then we have

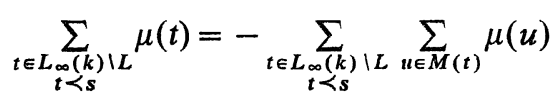

because of the assumption of our induction. Notice that

$$
\{u \in L(k-1) ; u \prec s\}=\underset{\substack{t \in L_{\infty}(k) \backslash L \\ t<s}}{\cup} M(t) \quad \text { (disjoint) }
$$

due to 1). Thus we have

$$
\sum_{\substack{t \in L_{\infty}(k) \backslash L \\ t<s}} \mu(t)=-\sum_{\substack{u \in L(k-1) \\ u \prec s}} \mu(u)
$$

by (4.2) and (4.3). Therefore we obtain

Finally we have

$$
\begin{aligned}
& \sum_{\substack{t \in L_{\infty}^{\prime} L \\
t \prec s \\
t \neq s}} \mu(t)=\sum_{k=0}^{i-1} \sum_{\substack{t \in L_{\infty}^{\infty}(k) \backslash L \\
t<s}} \mu(t) \\
& =-\sum_{k=0}^{i-1} \sum_{\substack{u \in L(k-1) \\
u \gtrless s}} \mu(u) \\
& =-\sum_{\substack{u \in L \\
r(u)<i-1 \\
u<s}} \mu(u) .
\end{aligned}
$$

$$
\begin{aligned}
\mu(s) & =-\sum_{\substack{t \in L_{\infty} \\
t \in s \\
t \neq s}} \mu(t) \\
& =-\sum_{t \in M(s)} \mu(t)-\sum_{\substack{u \in L \\
r(u)<i-1 \\
u<s}} \mu(u)-\sum_{\substack{t \in L_{\infty} \backslash L \\
t \geq s \\
t \neq s}} \mu(t) \\
& =-\sum_{t \in M(s)} \mu(t) .
\end{aligned}
$$

(4.4) Proposition.

$$
\sum_{s \in L(i)} \mu(s)-\sum_{s \in L(i-1)} \mu(s)=\sum_{s \in L_{\infty}(i)} \mu(s)
$$


for any integer $i<n+2$.

Proof.

$$
\begin{array}{rlrl}
\sum_{s \in L_{\infty}(i)} \mu(s)-\sum_{s \in L(i)} \mu(s) & =\sum_{s \in L_{\infty}(i) \backslash L} \mu(s) & \\
& =-\sum_{s \in L_{\infty}(i) \backslash L} \sum_{t \in M(s)} \mu(t) & & (\text { by }(4.1), 2)) \\
& =-\sum_{t \in L(i-1)} \mu(t) & & \text { (by (4.1), 1)). }
\end{array}
$$

Q.E.D.

We shall prove Theorem A as follows:

$$
\begin{array}{rlrl}
b_{i}\left(\mathbb{C}^{n+1} \backslash|X|\right) & =b_{i}\left(\boldsymbol{P}^{n+1} \backslash\left|X_{\infty}\right|\right) & \\
& =(-1)^{i} \sum_{j=0}^{i} \sum_{s \in L_{\infty}(j)} \mu(s) & & \text { (by (3.3)) } \\
& =(-1)^{i} \sum_{s \in L(i)} \mu(s) & & \text { (by (4.4)) }
\end{array}
$$

for $i<n+2$. If $i \geqq n+2$, then

$$
b_{i}\left(\boldsymbol{C}^{n+1} \backslash|X|\right)=0=(-1)^{i} \sum_{s \in L(i)} \mu(s)
$$

because $L(i)=\varnothing$

(4.5) Definition. An affine $n$-arrangement $X$ is said to be free if $X_{\infty}$ is a free projective $n$-arrangement. Let $X$ be free. Then the generalized exponents of $X$ are defined to be the generalized exponents of $X_{\infty}$.

Then this definition is consistent with the definition in Section 2.

Theorem B is immediately derived from Theorem B (projective version) and the very definition (4.5) of the generalized exponents of an affine $n$-arrangement.

\section{References}

[1] Orlik, P. and Solomon, L., Combinatorics and topology of complements of hyperplanes, Invent. Math., 56 (1980), 167-189.

[2] Terao, H., Forms with logarithmic pole and the filtration by the order of the pole, Proc. Intern. Sympo. on Algebraic Geometry, Kyoto, 1977, Kinokuniya, Tokyo, 1978, 673-685.

[3] -, Arrangements of hyperplanes and their freeness I, J. Fac. Sci. Univ. Tokyo Sect. IA Math., 27 (1980), 293-312.

[4] -._ - Arrangements of hyperplanes and their freeness II-the Coxeter equality-, ibid., 313-320.

[5] — Generalized exponents of a free arrangement of hyperplanes and ShepherdTodd-Brieskorn formula, Invent. Math., 63 (1981), 159-179. 
